
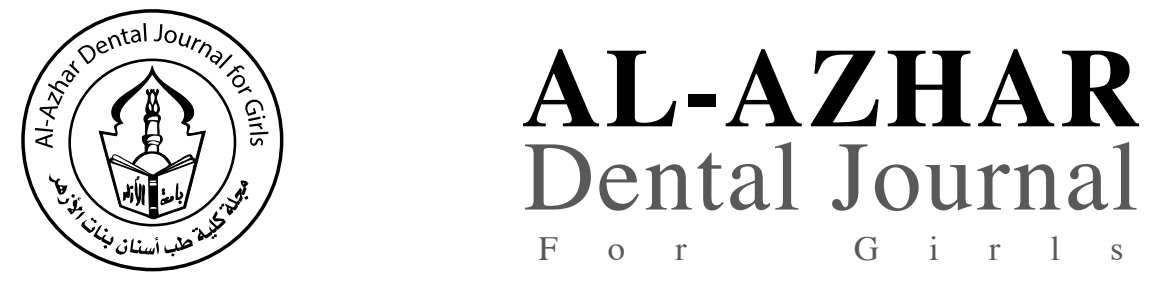

The Official Publication of The Faculty of Dental Medicine For Girls, Al-Azhar University Cairo, Egypt.

ADJ-for Grils, Vol. 4, No. 2, April (2017) — PP. 139:146

\title{
Evaluation of the Effect of Platelet Rich Fibrin on Alveolar Socket Healing in Glucocorticoids-Induced Osteoporosis in Rabbits (Histological and Immuno-histomorphometric study)
}

\author{
Heba Abd El-Fattah Zaki ${ }^{(1)}$, Mona HM Farid ${ }^{(2)}$ and Marwa AM El Shiekh ${ }^{(3)}$
}

Codex : 17/1704

dentaljournal.forgirls@yahoo.com
Paper extracted from master thesis entitled "Evaluation of the Effect of Platelet Rich Fibrin on Alveolar Socket Healing in GlucocorticoidsInduced Osteoporosis in Rabbits (Histological and Immunohistomorphometric study).

\begin{abstract}
Purpose: The purpose of this study was to evaluate the effect of platelet rich fibrin on the healing of osteoporotic alveolar socket after tooth extraction in experimentally induced osteoporosis in rabbits. Materials and Methods: Thirty six adult female rabbits were used in this study. Rabbits were divided into 3 groups (group1, group2 \&group 3: 12 for each group) then each group was divided into 3 subgroups (4 for each subgroups) according to the date of scarification at 7,20,30 days postoperatively. Group 1: (12 rabbits), alveolar socket was left empty after first molar extraction (didn't receive Platelet Rich Fibrin (PRF)). For the remaining 24 female rabbits: osteoporosis was experimentally induced by receiving $1.56 \mathrm{mg} / \mathrm{kg}$ dexamethasone (Dex) intraperitoneal injection daily for 2 months to induce osteoporosis - like condition. Group 2: (12 rabbits), osteoporotic alveolar sockets were left empty after first molar extraction (didn't receive PRF). Group 3: (12 rabbits), osteoporotic alveolar socket received PRF after first molar extraction. The specimens were collected, prepared and examined by routine haematoxylin \&eosin and immunostained by Osteoprotogrin (OPG) marker. Results: at 7,20 and 30 days, the greatest mean value was recorded in group 1, followed by group 3, with the least value recorded in group 2. Both group 1and 3 revealed a decrease in area percent of OPG throughout the study, whereas in group 2 revealed an increase. Conclusions: The study offers a clear evidence that topical application of PRF in osteoporotic alveolar socket after tooth extraction accelerates and increases the rate of bone formation and maturation than in osteoporotic alveolar socket without PRF.
\end{abstract}

\section{INTRODUCTION}

Osteoporosis is a global public health problem currently affecting more than 200 million people worldwide. Major researches efforts are being made to improve the outcome for patients with osteoporosis.
Platelet- rich fibrin $(P R F)$,

alveolar socket,

glucocorticoids (GCs),

Osteoporosis,

Osteoprotogrin $(O P G)$.

1. Demonstrator of Oral and Dental Biology, Oral and Dental Biology Department, Faculty of Dental Medicine, Al-Azhar University for Girls.

2. Professor and Head of Oral and Dental Biology Department, Faculty of Dental Medicine, Al-Azhar University for Girls.

3. Lecturer of Oral and Dental Biology, Oral and Dental Biology Department, Faculty of Dental Medicine, Al-Azhar University for Girls. 
It is characterized by loss of bone mass and deterioration of bone microarchitecture resulting in increased bone fragility and susceptibility to fracture. Drop in estrogen level following the menopause, increasing age, prolonged immobilization, chronic disease and use of certain medications can ultimately result in osteoporosis ${ }^{(1)}$.

Moreover, excess tobacco consumption, malnutrition, vitamin D deficiency and hypogonadism may play a role. In addition, chronic use of systemic and inhaled GCs also contributes to increase the risk of bone loss ${ }^{(2)}$. Synthetic GCs are used in a wide variety of disorders, including autoimmune, pulmonary and gastrointestinal diseases, as well as in patients with malignancies or following organ transplantation. Although the indications for GCs in these various conditions are clear, the use of GCs affect the skeleton of patients, which ultimately lead to osteoporosis ${ }^{(3)}$.

In dentistry, dental caries and periodontitis, which are inflammatory diseases, have been regarded as main causes of tooth loss. However, the number of studies suggesting a positive relationship between systemic osteoporosis and alveolar bone loss or tooth loss has been increasing annually ${ }^{(4)}$. Nowadays tooth extraction becomes more important in complicating odontological treatment; tooth extraction unavoidably has influence on bone resorption and changes of gingival contours. Further treatment may become more complex in using dental implants and common prosthetics ${ }^{(5)}$.

Clinical studies reported that approximately 50\% of the original alveolar bone width was reduced within the first 12 months after tooth removal ${ }^{(6)}$. Volumetric alterations of the alveolar ridge can be unfavorable for future endosseous implant placement and implant esthetics. Marginal alveolar bone ridge protection has influence in achieving optimal functional, aesthetic prosthesis and orthodontic treatment results. There is increasing demand in lowering damage to soft and hard tissues around the tooth being extracted. A traumatic tooth extraction and further protection of the alveolus is important ${ }^{(7)}$. So, using varieties of techniques for preservation of bone volume is of great importance to obtain good aesthetic results. Therefore, socket preservation has been recommended at time of tooth extraction to compensate for the postoperative volumetric alterations by using a grafting bone substitute as an example ${ }^{(8)}$.

PRF represents a new step in the platelet gel therapeutic concept with simplified processing excluding artificial biochemical modification. Unlike other platelet concentrates this technique requires neither anticoagulants nor bovine thrombin making it simple in its preparation, and considered as one of biological material that can promote osteogenesis in tooth extraction sockets and its effect is remarkable at the earlier time of bone formation ${ }^{(9)}$. Up right now, a complete preservation of the alveolar contour after tooth extraction with intra- socket grafts seems to be an unpredictable treatment goal ${ }^{(10)}$.

The purpose of the present study was to evaluate the effect of platelet rich fibrin on the healing of osteoporotic alveolar socket after tooth extraction in experimentally induced osteoporosis in rabbits.

\section{MATERIALS AND METHODS}

Thirty six adult female rabbits weighting an average of (2.5-3 kg) were used in this study. Rabbits were divided into 3 groups (12 for each group) then each group divided into 3 subgroups (4 for each subgroups) according to the date of scarification at 7, 20, 30 days postoperatively.

Group 1: (Negative control group) (12 rabbits) where alveolar sockets were left empty after first molar extraction (didn't receive PRF). Experimental groups: For the remaining 24 female rabbits, osteoporosis was experimentally induced by receiving $1.56 \mathrm{mg} / \mathrm{kg}$ Dex intraperitoneal injection daily for 2 months to induce osteoporosis - like condition ${ }^{(11)}$. The osteoporotic rabbits were divided into two groups 12 for each. 
Group 2: (Positive control group) (Osteoporotic group), osteoporotic alveolar sockets were left empty after first molar extraction (didn't receive PRF). Group 3: (Treatment group) (PRF group), osteoporotic alveolar sockets received PRF after first molar extraction.

\section{1) Preparation of PRF}

PRF was prepared in accordance with protocol developed by Choukroun intravenous blood was collected from marginal ear vein in Eppendorf sterile tube $2 \mathrm{ml}$, without anticoagulant and immediately centrifuged in centrifugation machine at 3,000 revolutions per minute for 10 minutes. PRF was easily separated from red corpuscles using a sterile tweezers.

Within few minutes, absence of anticoagulant allows activation of majority of platelets in the sample to start coagulation cascade. Initially fibrinogen is concentrated in the upper part of the tube until circulating thrombin transforms it into a fibrin network. The outcome is a fibrin clot (PRF) containing platelets in the middle of the tube between the red blood cell layer at the bottom and a cellular plasma at the top. This clot is removed from the tube and the attached red blood cells scraped off and discarded ${ }^{(12 \text { and 13). }}$

\section{2) Extraction}

The rabbits were anesthetized intramuscularly using $0.1 \mathrm{ml} / 200 \mathrm{~g}$ of animal weight using equal parts of ketamine and xylazine ${ }^{(14)}$.Then mandibular left first molar of each rabbit in all groups was extracted by using straight apexo and pynote forceps after 8 weeks from the beginning of drug administration. No antibiotic or other medications were used, then application of PRF inside the socket by condenser was done.

\section{3) Sample collection}

At the end of the experimental period for each subgroup, rabbit scarification was done by slaughter. Their heads were immediately dissected to obtain the mandibular molar area. The specimens were processed for routine histological and immunehistomorphometric analysis under light microscope.

\section{Evaluation of OPG immunostaining by image analysis}

The number of positively reacting immunostaining of OPG was counted. Counting was based on the area with the highest percentage of positive cells, each stained nucleus was regarded as positive regardless of the staining intensity. The sites expressing a positive immune reaction with the OPG monoclonal antibody are identified as brown deposits of the chromogen. The stronger the immune reaction, the darker the chromogen intensity. (Fig. 1 and 2 (A))

\section{RESULTS}

In (group 1) at 7 days, the socket was filled with granulation tissue and minute inflammatory cells. At the depth of the socket, small spicules of newly formed bone were observed. The bony spicules showed different degrees of stainability enclosing entrapped osteocytes. Multiple scattered hemorrhagic areas were clearly observed at the top of the socket. While in (group 2), the socket was completely filled with pronounced fibrous connective tissue where the collagen fibers appeared to be arranged in bundles having different courses and directions. Scattered minute bony spicules were observed. In (group 3), the socket showed multiple large interconnected bone spicules of variable size and shape. The enclosed osteocytes within those bony spicules lack normal architecture as their nuclei were eccentric and appeared with darkly basophilic stain. Negligible amounts of inflammatory cells were also noticed. (Fig.1(B)) 


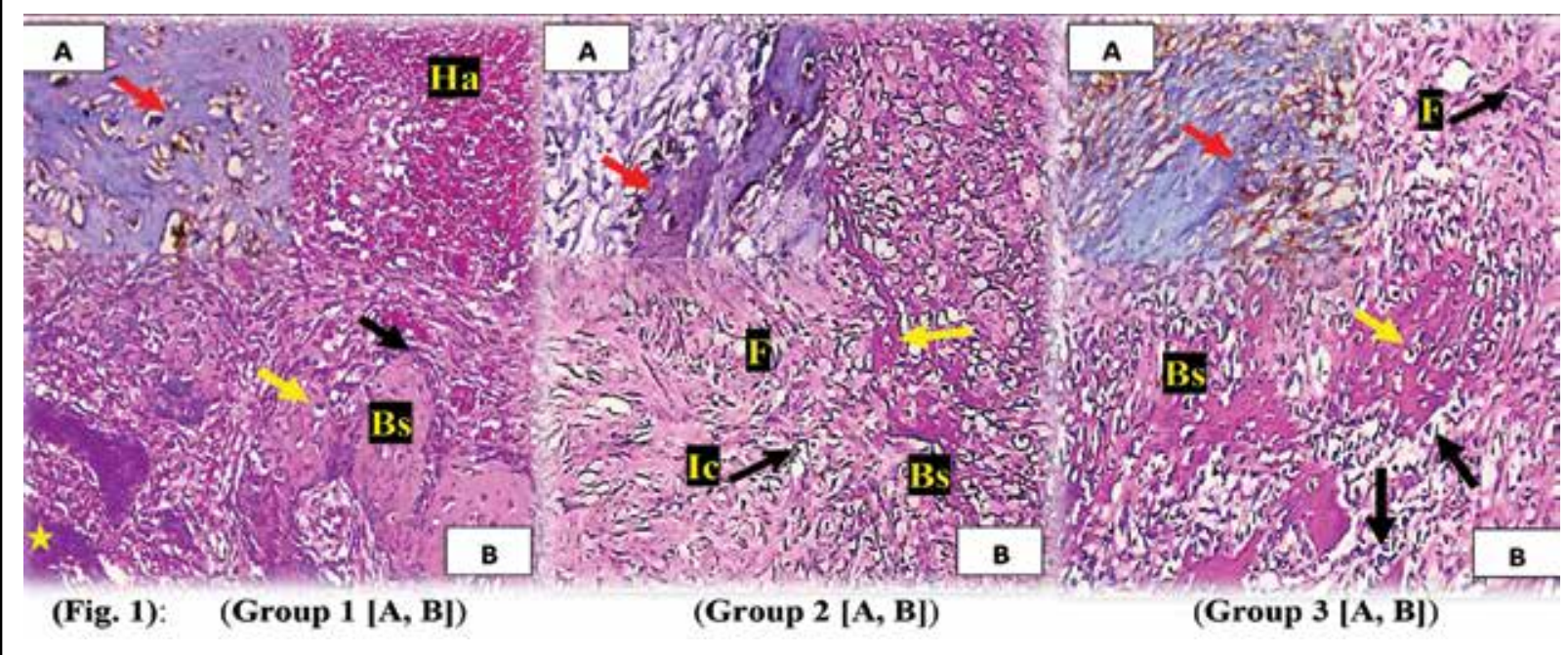

Fig. (1) BS (bone spicules), Ha (hemorrhagic area), F (fibers), Ic (inflammatory cells), Yellow arrow (osteocytes), Black arrow (osteoblasts), Red arrow (OPG stain osteocyte) and Star (different in degree of stainability of bone spicules.

In (group 1) at 20 days the socket was filled by variable sized and shaped bony spicules, with osteocytes of normal appearance \& arrangement enclosed inside. The periphery of those spicules was lined by osteoblasts. While in (group 2), little amount of thin rod -like bony spicules were seen, enclosing few number of osteocytes with wide lacunae and eccentric nuclei. The osteocytes were arranged in haphazard manner in some of these spicules while others appeared completely lacking the osteocytes. In (group 3), moderate amount of newly formed bone spicules were noticed, with many osteocytes entrapped inside and arranged in haphazard distribution and some of them show eccentric nuclei.

In (group 1) at 30 days the socket area was filled with compact bone which take the typical morphology of normal compact bone where many Haversian canals and Volkmann's canals containing blood vessels filled with R.B.Cs. were detected and surrounded by the normal architecture of osteocytes. While in (group 2), pronounced amount of bone trabeculae which appear interconnected, and enclosing different fibrous bone marrow spaces can be detected, with evidence of future formation of Haversian canal. In (group 3) good amount of bone formation with the relative normal architecture of bone trabeculae were seen, as number of osteocytes appeared arranged with relative normal shape and architecture (Fig. 2 (B)).

OPG was detected as a brown chromogen staining of tissue components expressing a positive OPG monoclonal antibody immune reactivity. The reaction appeared as brown deposits in cells, but was a homogenous brownish area in fluids and tissue ground substance. ${ }^{(15)}$

During light microscopic examination of immunohistological sections of dissected rabbits sections, positive OPG immune reaction could be localized in cells of osteocytes and osteoblasts of bony tissue of newly formed bone of the alveolar socket.

Both normal and PRF groups revealed a decrease in area percent of OPG throughout the study, whereas the osteoporosis group revealed an increase. Comparing the percent change in area percent of osteoproteogerin throughout the study in different groups, One way analysis of variance (ANOVA) test revealed that the difference of mean value was statistically significant $(\mathrm{p}=0.0013)$. Tukey's post hoc test revealed no significant difference between control and PRF groups. Significant at $p<0.05$. 


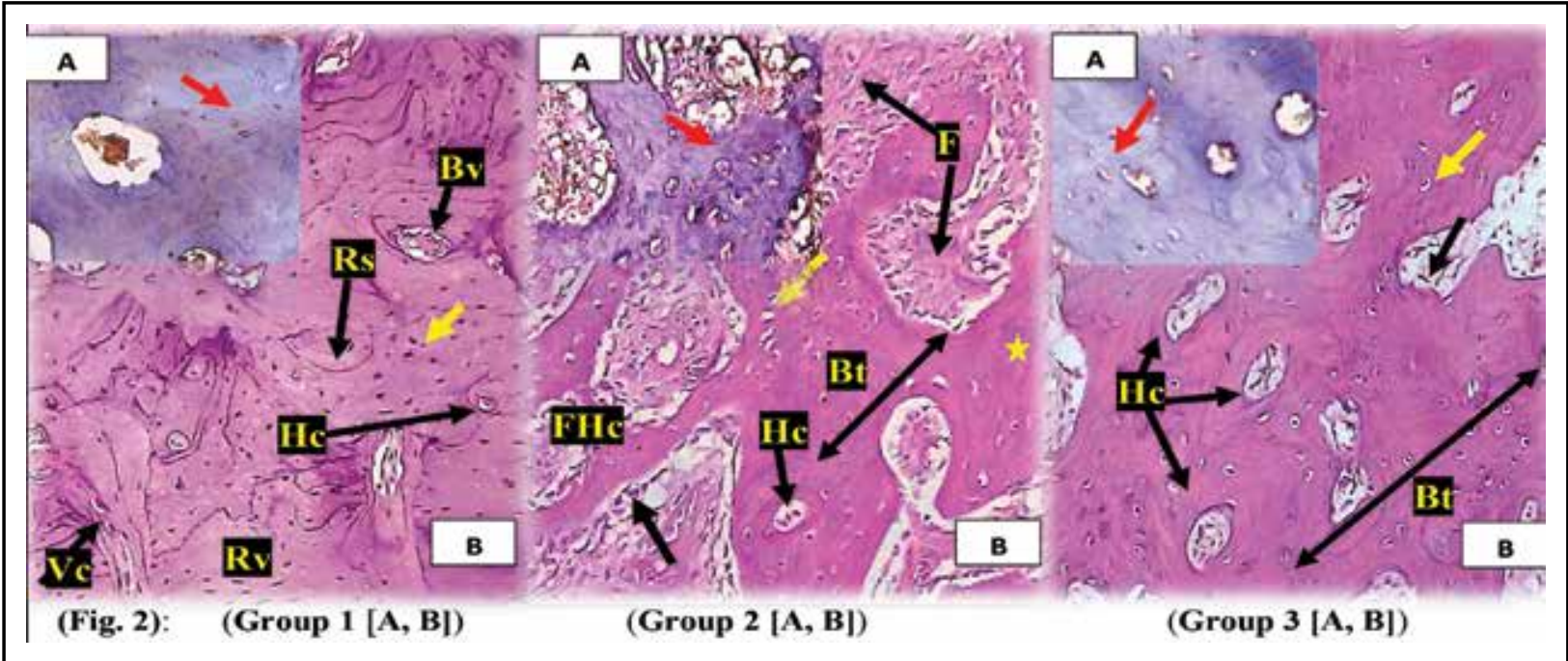

Fig. (2) Bt (bone trabeculea), Bv (blood vessel), F (fibers), Rs (resting line), Hc (Haversian canal), Vs (Volkmann's canal), Rv (reversal line), FHc (future Haversian canal), Yellow arrow (osteocytes) and Red arrow (OPG stain osteocyte).

\section{DISCUSSION}

Rabbits were animals of choice for being one of most commonly used animals for medical research, being used in approximately $35 \%$ of musculoskeletal research studies. This is due to ease of handling and size. Rabbits are also convenient in that it reaches skeletal maturity shortly after sexual maturity around 6 months of age ${ }^{(16)}$. It has an active Haversian remodeling, which might be very useful to test agents acting on bone remodeling, especially those with strong anabolic effects ${ }^{(17)}$.

Osteoporosis are induced experimentally through different methods ${ }^{(18-20)}$, one of main method to induce osteopetrosis is ovariectomy ${ }^{(21-26)}$ but it was found that rabbits constitutes a poor model for exclusively ovarian function deprivation osteoporosis. Failure in achieving significant reductions in bone mass of rabbit could be explained by presence of reflex ovulations or an ectopic production of sexual hormones ${ }^{(27)}$, so osteoporosis were induced in present study by using GCs.

These forgoing histologic events comprising tooth extraction socket healing have been described in details ${ }^{(28,29, \text { and } 30)}$. the collecting specimens from group 2 (osteoporotic group) at 7 days showed delay in bone healing related to Dex administration which lead to reduction in bone formation and increase in bone resorption where socket still filled with collagen fiber and negligible amounts of bony spicules was noticed along the periphery. These histological findings were in agreement with the researchers ${ }^{(31,32)}$ who found that Dex reduce expression of insulinlike growth factors I and II as this growth factors known to increase osteoblasts differentiation, type I collagen synthesis and bone formation.

The histological findings at 20 and 30 days illustrate little amount of thin rod -like structure, sparse and randomly oriented bone trabeculae especially at the cervical and middle part of the extracted socket. This histological finding was in agreement with other researchers who stated that, ${ }^{(33,34)}$ Dex decreased blood vessels and decreased mineralizing surfaces by decreasing bone formation through suppressing osteoblastic activity and function in bone marrow. Furthermore, Dex increase osteoblasts and osteocytes apoptosis and cause heterogeneity of bone loss.

The immuno-histomorphometric results was concomitant with the histological results as in group 2 (osteoporotic group) which revealed that, 
when OPG stained osteoblasts and osteocytes the lowest mean value of OPG was recorded at 7 days, with a slight increase of OPG stain in osteoblasts and osteocytes at 20 and 30 days. This due to the action of Dex administration, which delay healing of extracted sockets by increasing osteoblasts and osteocytes apoptosis and affect expressions of osteoblasts and osteocytes to OPG which considered as a bone protector as it protects the skeleton from excessive bone resorption ${ }^{(35)}$. (Fig. 1 and 2 (A)).

Comparing the histological results observed at 7 days in both group 1 (control group) and group 2 (osteoporotic group) it was found that in groups treated with PRF (group 3), formation of bone spicules with active osteoblast and dilated blood vessels engorged with R.B.Cs. while in group 2 (osteoporotic group) only primitive bone formation in the alveolar socket was formed. This is in agreement with study done by investigator ${ }^{(36)}$ who found that platelets can enhance plasminogen activation capacity of mesenchymal progenitors which responsible for bone formative cell.

At 20 days postoperatively, at this healing period, the histological findings illustrate, bone trabeculae formation in all groups, furthermore, delay of bone formation was very obvious in extracted socket of experimental group without PRF group 2 (osteoporotic group) by presence of fibrous connective tissue in some area, while in extracted socket with PRF (group 3) showed new bone formation with large number of osteocytes, many reversal and resting lines, and also with Haversian canal formation. These histological findings was in agree with investigator ${ }^{(37)}$ who found that PRF has an osteo-promotive activity since it contains a high concentration of growth factors, such as transforming growth factor - beta 1,2 and vascular endothelial growth factor. These growth factors promote regeneration of blood vessel as well as proliferation and migration of osteoblasts.

At 30 days postoperatively, in comparing between group 2 (osteoporotic group) and group 3
(PRF group), application of PRF to the extracted socket had a positive effect on the healing process as good amount of bone formation with relative normal architecture of bone trabeculae was observed with presence of relatively normal Haversian canals in group 3 (PRF group), These findings indicate that the platelets within PRF release growth factors and proteins like osteonectin, fibronectin, and osteocalcin , all of them influence bone healing in different ways ${ }^{(38)}$

Comparing the results of group 1 (control group), group 2 (osteoporotic group) and group 3 (PRF group). As measured by ANOVA it was found that the greatest mean value of immuno- expression of OPG was recorded at 7 days in group 1 (control group), followed by 6 group 3 (PRF group), while the least value recorded in group 2 (osteoporotic group). As a result of apoptosis of osteoblastlineage cells evoking a decrease in release of OPG by these cells.

These foregoing findings was in agreement with other studies ${ }^{(39)}$, which found that the alveolar repair process associated with the receptor activator of NF- $x \mathrm{~B}$ ligand and OPG pattern being inversely proportional. As there was an increase in bone formation during the course of the analyzed periods, while there was a reduction in the expression of these proteins, indicating complete filling of the alveolar process associated with a balance in bone turnover.

\section{CONCLUSIONS}

1. The present study offers a clear evidence that topical application of PRF in osteoporotic alveolar socket after tooth extraction accelerate and increase rate of bone formation and maturation than in osteoporotic alveolar socket without PRF.

2. Ease of preparation/application and lack of biochemical modification by utilizing the patient's own blood reduces or eliminates disease transmission through blood. 
3. Easily applied PRF acts much like a fibrin bandage, serving as a matrix to accelerate the healing of wound. Also acts as a biological connector between the bone particles.

4. The intrinsic incorporation of cytokines within the fibrin mesh allows for their progressive release over time (7-11 days), as the network of fibrin disintegrate.

\section{REFERENCES}

1. Luo M, Zheng M, Lei H, Zhang S, Li Y. Association between bone morphogenetic protein 2 polymorphisms and osteoporotic fracture. Int J Clin Exp Pathol 2016; 9(2):1961-7.

2. Franco C, Paz G, Gomes P, Nascimento V, Kulak C. Chronic obstructive pulmonary disease is associated with osteoporosis and low levels of vitamin D. Osteoporis Int 2009; 20(11):1881-7.

3. Sun P, Cai D, Li Q, Chen H, Deng W. Effects of alendronate and strontium ranelate on cancellous and cortical bone mass in glucocorticoid-treated adult rats. Calcif Tissue Int 2010; 86(6):495-501.

4. Wowern Nv, Klausen B, Kollerup G. Osteoporosis: a risk factor in periodontal disease. J Periodontol 1994; 65(12):1134-8.

5. Cheng LL. Alveolar ridge preservation with bone graft may limit physiological ridge loss after tooth extraction. $\mathrm{J}$ Am Dent Assoc 2016; 147(3):204-6.

6. Aggarwal G, Iyer N, Bali A, Popli G, Malhotra A. Comparative evaluation of success and survival rates of two piece implant placed immediately or delayed into extraction site: A clinical-radiological study. J Oral Maxillofac Surg 2016; 3(1):1-9.

7. Kubilius M, Kubilius R, Gleiznys A. The preservation of alveolar bone ridge during tooth extraction. Stomatologija 2012; 14(1):3-11.

8. Whetman $\mathrm{J}$ and Mealey BL. Effect of healing time on new bone formation following tooth extraction and ridge preservation with demineralized freeze-dried bone allograft. A randomized controlled clinical trial. J Periodontol 2016; 1(2):1-13.

9. Gawai KT and Sobhana C. Clinical evaluation of use of platelet rich plasma in bone healing. J Oral Maxillofac Surg. 2015; 14(1):67-80.
10. Fickl S, Zuhr O, Wachtel H, Stappert CF, Stein JM. Dimensional changes of the alveolar ridge contour after different socket preservation techniques. J Clin Periodontol 2008; 35(10):906-13.

11. Yasear A and Hamouda S. Effect of dexamethasone on osteoclast formation in the alveolar bone of rabbits. Iraqi $\mathrm{J}$ Vet Sci 2009; 23(1):13-6.

12. Singh A, Kohli M, Gupta N. Platelet rich fibrin: a novel approach for osseous regeneration. J Oral Maxillofac Surg 2012; 11(4):430-4.

13. Prakash S and Thakur A. Platelet concentrates: past, present and future. J Oral Maxillofac Surg. 2011; 10(1):45-9.

14. Cantín M, Olate S, Fuentes R, Vasquez B. Alveolar ridge conservation by early bone formation after tooth extraction in rabbits. A histomorphological study. Int $\mathbf{J}$ Morphol 2015; 33(1):369-74.

15. Shewel Y, Morad G, Bassiouny M, Ebaeid T. Immunohistochemical study of the possible role of osteoprotegerin OPG in inhibition of otic capsule remodeling. Egy. J. ear, nose, throat and allied sci. 2015; $16(1): 47-53$.

16. Pearce A, Richards R, Milz S, Schneider E, Pearce S. Animal models for implant biomaterial research in bone: a review. Eur Cell Mater 2007; 13(1):1-10.

17. Baofeng L, Zhi Y, Bei C, Guolin M, Qingshui Y. Characterization of a rabbit osteoporosis model induced by ovariectomy and glucocorticoid. Acta Orthop 2010; 81(3):396-401.

18. Simann M, Schneider V, Le Blanc S, Dotterweich J, Zehe V. Heparin affects human bone marrow stromal cell fate: promoting osteogenic and reducing adipogenic differentiation and conversion. Bone 2015; 78(6):102-13.

19. Castiglioni S, Cazzaniga A, Albisetti W, Maier JA. Magnesium and osteoporosis: current state of knowledge and future research directions. Nutrients 2013; 5(8):3022-33.

20. Song L, Xie XB, Peng LK, Yu SJ, Peng YT. Mechanism and treatment strategy of osteoporosis after transplantation Int J Endocrinol Metab Disord 2015; 1(3):15-20.

21. Aras MH, Bozdag Z, Demir T, Oksayan R, Yanık S. Effects of low-level laser therapy on changes in inflammation and in the activity of osteoblasts in the expanded premaxillary suture in an ovariectomized rat model. Photomed Laser Surg 2015; 33(3):136-44. 
22. Allen MR. Animal models of medication-related osteonecrosis of the jaw. Medication- related osteonecrosis of the jaws: Springer 2015; 3(1): 155-67.

23. Sigrist IM, Gerhardt C, Alini M, Schneider E, Egermann M. The long-term effects of ovariectomy on bone metabolism in sheep. J Bone Miner Metab 2007; 25(1):28-35.

24. Reinwald S and Burr D. Review of non-primate, large animal models for osteoporosis research. J Bone Miner Res 2008; 23(9):1353-68.

25. Dehoux JP and Gianello P. 1. Abstract 2. Introduction 3. Ethical considerations 4 . The importance of large animal models for transplantation research 5. Animal models. Front Biosci 2007; 12(1):4864-80.

26. Castaneda S, Calvo E, Largo R, Gonzalez R, De la Piedra C. Characterization of a new experimental model of osteoporosis in rabbits. J Bone Miner Metab 2008; 26(1):53-9

27. Mavropoulos A, Rizzoli R, Ammann P. Different responsiveness of alveolar and tibial bone to bone loss stimuli. J Bone Miner Res 2007; 22(3):403-10.

28. Amler MH. The time sequence of tissue regeneration in human extraction wounds. Oral Surg. Oral Med. Oral Pathol 1969; 27(3):309-18.

29. Guglielmotti M and Cabrini R. Alveolar wound healing and ridge remodeling after tooth extraction in the rat: a histologic, radiographic, and histometric study. J Oral Maxillofac Surg 1985; 43(5):359-64.

30. Huebsch RF and Hansen LS. A histopathologic study of extraction wounds in dogs. Oral Surg. Oral Med. Oral Pathol. 1969; 28(2):187-96.

31. Bouvard B, Legrand E, Audran M, Chappard D.
Glucocorticoid-induced osteoporosis: a review. Clin Rev Bone Miner Metab 2010; 8(1):15-26.

32. Guanabens N, Gifre L, Peris P. The role of wnt signaling and sclerostin in the pathogenesis of glucocorticoid-induced osteoporosis. Curr Osteoporos Rep 2014; 12(1):90-7.

33. Silverman SL and Lane NE. Glucocorticoid-induced osteoporosis. Curr Osteoporos Rep 2009; 7(1):23-6.

34. Moutsatsou P, Kassi E, Papavassiliou AG. Glucocorticoid receptor signaling in bone cells. Trends Mol Med 2012; 18(6):348-59.

35. Hofbauer LC, Khosla S, Dunstan CR, Lacey DL, Boyle WJ, Riggs BL. The roles of osteoprotegerin and osteoprotegerin ligand in the paracrine regulation of bone resorption. J Bone Miner Res 2000; 15(1):2-12.

36. Agis H, Kandler B, Fischer MB, Watzek G, Gruber R. Activated platelets increase fibrinolysis of mesenchymal progenitor cells. J Orthop Res 2009; 27(7):972-80.

37. Pei T, Nie R, Yang G, Che Y, Zhang D. A Comparative study of early bone formation with PRF, bio-oss and osteoid hydroxyapatite after tooth extraction in rabbits. $\mathrm{J}$ Hard Tissue Biol 2015; 24(1):29-36.

38. Kurikchy MQ, Al-Rawi NH, Ayoub RS, Mohammed SS Histological evaluation of bone healing using organic bovine bone in combination with platelet-rich fibrin (an experimental study on rabbits). Clin Oral Investig 2013; 17(3):897-904.

39. Luvizuto ER, Queiroz TP, Dias SMD, Okamoto T, Dornelles RCM. Histomorphometric analysis and immunolocalization of RANKL and OPG during the alveolar healing process in female ovariectomized rats treated with estrogen or raloxifene. Arch Oral Biol. 2010; 55(1):529. 